\title{
Sirt3 promotes the autophagy of HK-2 human proximal tubular epithelial cells via the inhibition of Notch-1/Hes-1 signaling
}

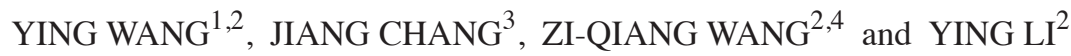 \\ ${ }^{1}$ Department of Nephrology, Bayan Nur Hospital, Bayan Nur, Inner Mongolia Autonomous Region 015000; \\ ${ }^{2}$ Department of Nephrology, The Third Hospital of Hebei Medical University, Shijiazhuang, Hebei 050051; \\ Departments of ${ }^{3}$ Hepatobiliary and Pancreatic Surgery and ${ }^{4}$ Blood Purification, \\ The First Affiliated Hospital of Hainan Medical College, Haikou, Hainan 570102, P.R. China
}

Received February 23, 2021; Accepted June 11, 2021

DOI: $10.3892 / \mathrm{mmr} .2021 .12273$

\begin{abstract}
Diabetic nephropathy (DN) is a predominant cause of end-stage renal disease. The impairment of the autophagy of human renal tubular epithelial cells (HK-2 cells) is involved in the pathogenic mechanisms of DN. Sirtuin (Sirt) 3 regulates the scavenging of damaged organelles and maintains energy balance. The present study aimed to examine the protective effects of Sirt3 on HK-2 cells stimulated by high glucose (HG). HK-2 cells were cultured in normal glucose (NG), HG or hyperosmotic medium. The viability of the HK-2 cells was detected using a Cell Counting Kit- 8 assay. The expression and localization of Sirt3 were detected via immunofluorescence. Following transfection with an overexpression plasmid, the expression levels of key components in the Notch homolog 1 (Notch-1)/hairy and enhancer of split-1 (Hes-1) pathway and those of the autophagy-related proteins, Beclin-1, LC-3II and p62, were measured by western blot analysis and reverse transcription-quantitative PCR (RT-qPCR). As the Notch-1/Hes-1 pathway was inhibited, the expression levels of Beclin-1, LC-3II and p62 were also examined at transcriptional and translational level. It was found that prolonged culture in HG medium markedly reduced cell viability compared with the cells cultured in NG or in NG + mannitol, an effect that was aggravated with the increasing duration of culture. HG was capable of inhibiting the expression levels of Beclin-1, LC-3II and Sirt3, and upregulating p62 and the Notch-1/Hes-1 pathway, as verified by western blot analysis and RT-qPCR. The results of immunofluorescence staining revealed that $\mathrm{HG}$ decreased Sirt3 expression. Sirt3 reversed the HG-induced inhibition of the expression of Beclin-1 and LC-3II and the upregulation of p62. Moreover, Sirt3 reversed the HG-induced inhibition of the Notch-1/Hes-1 signaling pathway. However,
\end{abstract}

Correspondence to: Dr Ying Li, Department of Nephrology, The Third Hospital of Hebei Medical University, 139 Ziqiang Road, Shijiazhuang, Hebei 050051, P.R. China

E-mail: yingliihebei@163.com

Key words: glucose, autophagy, Sirtuin 3, Notch homolog 1 this autophagy-promoting effect of Sirt3 was counteracted by the Notch-1/Hes-1 pathway activator. On the whole, the present study demonstrated that Sirt3 promoted the autophagy of HK-2 cells, at least partly, via the downregulation of Notch-1/Hes-1.

\section{Introduction}

As one of the severe microvascular complications of type 2 diabetes mellitus, diabetic nephropathy (DN) is the leading cause of end-stage renal disease (1). Although diagnosis and treatment technologies have improved, the incidence of DN is increasing worldwide (2). Therefore, there is an urgent need to clarify the molecular pathogenesis of $\mathrm{DN}$ and to identify therapeutic targets.

Autophagic dysfunction is considered to be involved in DN (3). Autophagy is a cellular recycling pathway that is essential for maintaining cellular integrity (3). Under stress conditions, human proximal tubular epithelial (HK-2) cells exploit the autophagic machinery to clear damaged organelles and protein aggregates. The degradation and recycling process of autophagy contributes to maintaining normal cellular functions and delays the progression of diabetic renal injury (4). Previous research has demonstrated that during autophagy, lysosomes contribute to the regulation of the degradation of damaged cellular components as an adaptive response, playing an important role in the pathogenesis of DN $(3,5)$. As a result, the high glucose (HG)-induced impairment of autophagy in renal tubular epithelial cells has emerged as an important process responsible for the occurrence and development of diabetic renal injury.

Multiple regulatory mechanisms modulate autophagy in DN, including nutrient signaling pathways (6). Among these, Sirtuin (Sirt)1 functions as an important regulator of autophagy (7). As a member of the Sirt family, Sirt1 has been reported to contribute to the clearance of damaged organelles and the maintenance of energy homeostasis (8). Sirt3 belongs to the same family as Sirt1, and is capable of regulating signaling pathways when stress occurs, thereby inducing autophagy $(9,10)$. In a previous study, in a model of sepsis-induced acute kidney injury, Sirt3 was demonstrated to promote autophagy, alleviating acute kidney injury, tubular cell apoptosis and inflammatory responses in the kidneys (11). 
Moreover, through the activation of the process of autophagy, Sirt3 significantly attenuates the occurrence and development of diabetes-related microvascular complications (12). Sirt3 has also been shown to inhibit mitochondrial injury and cardiomyocyte apoptosis through the activation of autophagy and mitophagy in HG-stimulated cardiomyocytes (13). Mesenchymal stem cells have been shown to attenuate diabetic lung fibrosis by modulating Sirt3-mediated stress responses, such as the enhancement of autophagy and inhibition of oxidative stress, inflammation, apoptosis and endoplasmic reticulum stress (14).

With regards to the pathways regulating autophagy, Notch homolog 1 (Notch-1)/hairy and enhancer of split-1 (Hes-1) has been proven to be involved in cell proliferation, differentiation and death (15). Moreover, this pathway has been shown to regulate the equilibrium between autophagosome formation and clearance by lysosomes in podocytes (16). However, whether this pathway plays a role in the autophagic process in renal tubular epithelial cells is not yet fully understood, and the function of Sirt3 in modulating autophagy in HG-stimulated proximal tubular epithelial cell injury remains undetermined. Thus, the present study aimed to evaluate the renoprotective effects of Sirt3 against DN. Additionally, whether Sirt3 overexpression attenuates kidney injury via modulating autophagy in HG-stimulated proximal tubular epithelial cells was determined.

\section{Materials and methods}

Cell culture and treatment. HK-2 cells were purchased from the American Type Culture Collection and cultured as previously described (17). All cells were cultured in DMEM (Invitrogen; Thermo Fisher Scientific, Inc.) with 10\% FBS (HyClone; Cygtiva) at $37^{\circ} \mathrm{C}, 5 \% \mathrm{CO}_{2}$ in a humidified incubator. Cells $\left(5 \times 10^{5}\right.$ cells/well) were seeded in 6 -well plates at $80 \%$ confluency and continued to be cultured in DMEM with $2 \%$ FBS. The HK-2 cells were serum-starved for $24 \mathrm{~h}$, followed by exposure to DMEM with $5.5 \mathrm{mM}$ glucose [normal glucose (NG)], $30 \mathrm{mM}$ glucose $(\mathrm{HG})$ or $\mathrm{NG}+$ hyperosmotic medium [24.5 mmol/1 mannitol (M)]. Following washing three times with 1X PBS, the HK-2 cells were harvested for the cell viability assay. This was followed by transfection with overexpression plasmid and the inhibition of the Notch-1/Hes-1 pathway.

Cell viability assay. HK-2 cells were cultured in the designated medium for an additional 6, 12, 24, 48 and $72 \mathrm{~h}$. Cell viability was determined using a Cell Counting Kit-8 (CCK-8; MedChemExpress) assay. Briefly, the HK-2 cells were washed and plated at a density of $1 \times 10^{3}-10^{4}$ cells per well in a 96-well plate. Cells were incubated in fresh medium at $37^{\circ} \mathrm{C}$ for an additional 48 h. Subsequently, CCK-8 solution (10 $\mu \mathrm{l})$ was added to each well of the culture medium at each time point, according to the manufacturer's instructions. The plates were kept at $37^{\circ} \mathrm{C}$ with $5 \% \mathrm{CO}_{2}$ in an incubated plate holder for $1 \mathrm{~h}$. The absorbance was determined using a microplate reader (BioTek microplate reader; BioTek Instruments, Inc.) at $450 \mathrm{~nm}$.

Transfection with overexpression plasmid. HK-2 cells were seeded in a 6 -well plate at a density of $5 \times 10^{5}$ cells/well for
48 h. Plasmid transfection was performed using FuGENE 6 (Promega Corporation) according to the manufacturer's instructions. The plasmids used in the present study were described previously and confirmed by DNA sequencing (18). Cells were transfected with $2 \mu \mathrm{g}$ pCMV-SIRT3 or empty pCMV-vector (Integrated Biotech Solutions) at $37^{\circ} \mathrm{C}$. Following $48 \mathrm{~h}$ of transfection, the HK-2 cells were stimulated with $\mathrm{HG}$ for $48 \mathrm{~h}$ at $37^{\circ} \mathrm{C}$. The transfection efficacy was determined by reverse transcription-quantitative PCR (RT-qPCR) and western blot analysis.

Inhibition of the Notch-1/Hes-1 pathway. To activate the Notch-1/Hes-1 pathway, Jagged1-FC (final concentration, $0.5 \mu \mathrm{g} / \mathrm{ml}$; cat. no. $1277-\mathrm{JG}$; R\&D Systems, Inc.) was added to the medium and the incubation was continued for a further $2 \mathrm{~h}$ at room temperature.

Western blot analysis. Western blot analysis was performed as previously described (19). The HK-2 cells were washed with 1X PBS three times and lysed with RIPA lysis buffer (Beyotime Institute of Biotechnology). Total protein extracts were collected and the concentration was quantified using a BCA assay (Pierce; Thermo Fisher Scientific, Inc.). Total protein $(30 \mu \mathrm{g})$ was loaded onto $10 \%$ SDS-PAGE (Beijing Solarbio Science \& Technology Co., Ltd.) followed by transfer onto PVDF membranes (Bio-Rad Laboratories, Inc.). The membranes were blocked with TBS containing $0.1 \%$ Tween-20 (TBST) containing 5\% non-fat milk for $1 \mathrm{~h}$ at room temperature, followed by primary antibody incubation overnight at $4^{\circ} \mathrm{C}$. The antibodies were as follows: Anti-Beclin-1 (cat. no. ab210498; 1:1,000; Abcam), anti-LC-3II (cat. no. ab48394; 1:1,000; Abcam), anti-p62 (cat. no. ab56416; 1:1,000; Abcam), anti-Sirt3 (cat. no. ab217319; 1:1,000; Abcam), anti-Notch-1 (cat. no. ab52627; 1:1,000; Abcam), anti-Hes-1 (cat. no. ab108937; 1:1,000; Abcam) and anti- $\beta$-actin (cat. no. ab8227; 1:1,000; Abcam). Following washing with TBST three times for $15 \mathrm{~min}$, the membranes were then incubated with HRP-conjugated secondary antibodies (cat. no. 7074 and 7076; 1:1,000; Cell Signaling Technology, Inc.) for $2 \mathrm{~h}$ at room temperature. Proteins were visualized using the ECL method (Thermo Fisher Scientific, Inc.). Semi-quantification of the western blot bands was performed using ImageJ software (version 1.8.0; National Institutes of Health).

$R T-q P C R$ analysis. Total cellular mRNA of Beclin-1, LC-3II, p62, Sirt3, Notch-1 and Hes-1 was isolated using TRIzol ${ }^{\circledR}$ reagent (Invitrogen; Thermo Fisher Scientific, Inc.), according to the manufacturer's instructions. RT-qPCR was performed as previously described (15). Total RNA $(2 \mu \mathrm{g})$ extracted from the cultured cells was used as a template for cDNA synthesis. The primer sequences were as follows: Beclin-1 forward, 5'-AAT GACTTTTTTCCTTAGGGGG-3' and reverse, 5'-GTGGCT TTTGTGGATTTTTTCT-3'; LC-3II forward, 5'-AGTGCC TGTGTTGTTACGGA-3' and reverse, 5'-GCAGAAGGGAGT GTGTCTGA-3'; p62 forward, 5'-AGTCGGAGCGGGTTC TCTAT-3' and reverse, 5'-GTGACACACATTCCAGCGAT-3'; Notch-1 forward, 5'-CAGACAGGCAGGTGGGGTCGTGGT A-3' and reverse, 5'-GCGACAACGCCTACCTCTG-3'; Hes-1 forward, 5'-CAACACGACACCGGATAAAC-3' and reverse, 


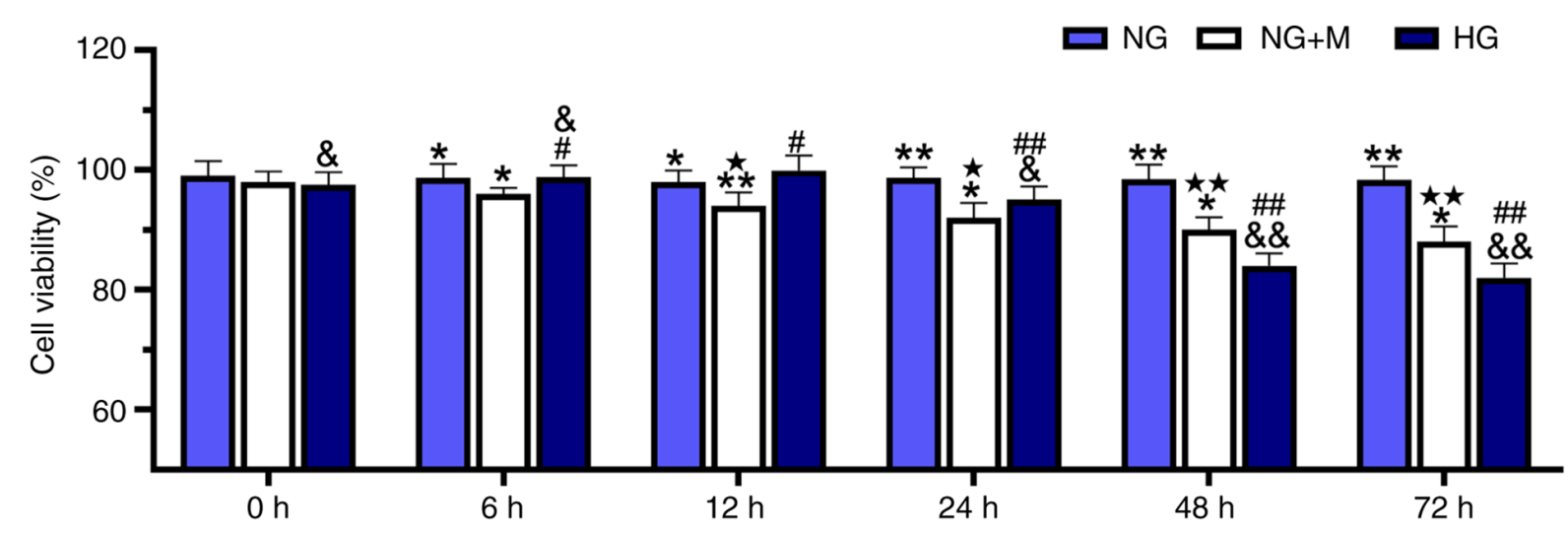

Figure 1. HG reduces the viability of HK-2 cells. Values are presented as the mean $\pm \mathrm{SD}$. ${ }^{*} \mathrm{P}<0.05$ and ${ }^{* *} \mathrm{P}<0.01 \mathrm{vs}$. HG; ${ }^{*} \mathrm{P}<0.05$ and ${ }^{\# \#} \mathrm{P}<0.01$ vs. HG at 0 h; ${ }^{*} \mathrm{P}<0.05$ and ${ }^{* *} \mathrm{P}<0.01$ vs. $\mathrm{NG}+\mathrm{M}$ at $0 \mathrm{~h} ;{ }^{\&} \mathrm{P}<0.05$ and ${ }^{\&} \& \mathrm{P}<0.01$ vs. $\mathrm{HG}$ at $12 \mathrm{~h}$. NG, normal glucose (5.5 mM glucose); $\mathrm{M}$, mannitol (5.5 mM glucose + $24.5 \mathrm{mmol} / \mathrm{l}$ mannitol); HG, high glucose (30 mM glucose).

5'-TTCAGCTGGCTCAGACTTTC-3'; and $\beta$-actin forward, 5'-TGACGTGGACATCCGCAAAG-3' and reverse, 5'-CTG GAAGGTGGACAGCGAGG-3'. The conditions for amplification were as follows: Initial denaturation at $95^{\circ} \mathrm{C}$ for $5 \mathrm{~min}$, followed by 40 cycles each at $95^{\circ} \mathrm{C}$ for $10 \mathrm{sec}$, followed by $60^{\circ} \mathrm{C}$ for $30 \mathrm{sec}$, and $72^{\circ} \mathrm{C}$ for $45 \mathrm{sec} . \beta$-actin mRNA served as an endogenous control. The relative quantities of products were determined using the $2^{-\Delta \Delta C q}$ method (20). The experiments were performed in triplicate for each group.

Cell immunofluorescence. Following seeding on coverslips in 6-well plates $\left(2 \times 10^{5}\right.$ cells/well), the cells were fixed with $4 \%$ paraformaldehyde for $10 \mathrm{~min}$ at $4^{\circ} \mathrm{C}$. Subsequently, the cells were blocked with $10 \%$ goat serum (Beijing Solarbio Science \& Technology Co., Ltd.) for $15 \mathrm{~min}$ at room temperature, and subjected to immunofluorescence staining with anti-Sirt3 polyclonal antibody (1:500; cat. no. ab40963; Abcam) as the primary antibody. Following incubation overnight with anti-Sirt 3 antibody at $4^{\circ} \mathrm{C}$, the HK-2 cells were then incubated with Cy3-labeled IgG (cat. no. TA130020; 1:100; OriGene Technologies, Inc.) for $1 \mathrm{~h}$ at room temperature. The following day, the nuclei were stained with DAPI for $10 \mathrm{~min}$ at room temperature. Immunofluorescence was observed and captured using a fluorescence microscope (Olympus Corporation).

Statistical analysis. All experiments were carried out in triplicate. The analyses were performed using SPSS software (version 23.0; IBM Corp.). Data are expressed as the mean \pm SD. For multiple comparisons between groups, a one-way ANOVA followed by Tukey's post hoc test was performed. $\mathrm{P}<0.05$ was considered to indicate a statistically significant difference.

\section{Results}

$H G$ reduces the viability of $H K-2$ cells. HK-2 cell cultivation was performed in NG, HG or a hyperosmotic environment, and cell viability was then determined using a CCK- 8 assay (Fig. 1). When the HK-2 cells were incubated in HG culture medium for 6 and $12 \mathrm{~h}$, it was observed that there was a slight increase in cell viability compared with cells cultivated in NG. However, treatment with NG $+\mathrm{M}$ decreased cell viability compared with the NG group over the culturing time. Of note,
HG markedly decreased cell viability compared with the cells treated with $\mathrm{NG}$ and $\mathrm{NG}+\mathrm{M}$ with the duration of culture.

Long-term culture of HK-2 cells in HG inhibits the expression of Sirt3 and autophagy. After the cells were subjected to the different treatments ( $\mathrm{NG}, \mathrm{NG}+\mathrm{M}$ and $\mathrm{HG}$ ), the expression level of Sirt3 was detected (Fig. 2A). The expression of Sirt3 exhibited an unremarkable change in the HK-2 cells cultured in $\mathrm{NG}+\mathrm{M}$ compared with the NG group. Compared with the NG group, the protein expression level of Sirt3 was significantly decreased in the HK-2 cells following stimulation with HG for $48 \mathrm{~h}$ (Fig. 2B). A similar trend was observed in the cell immunofluorescence assay (Fig. 2J). Short-term HG culture $(6$ and $12 \mathrm{~h}$ ) led to a significant elevation in Sirt3 protein expression. However, the expression level of Sirt3 tended to decrease with the increasing incubation time. No further changes in Sirt3 protein expression were observed at $48 \mathrm{~h}$. Sirt 3 mRNA expression exhibited a similar trend with that of its protein expression determined by western blot analysis (Fig. 2C).

In order to evaluate the effects of $\mathrm{HG}$ on autophagic activation in HK-2 cells, western blot analysis and RT-qPCR were performed to determine the mRNA and protein expression levels of Beclin-1, LC-3II and p62 (Fig. 2A and D-I). HG increased the protein expression levels of Beclin-1 and LC-3II at 6 and $12 \mathrm{~h}$ compared with the NG group. However, at later time points, HG significantly decreased Beclin-1 and LC-3II protein expression levels. These levels exhibited a downward trend from $24 \mathrm{~h}$, with the most significant decrease observed at $48 \mathrm{~h}$ (Fig. 2D and F). With regards to p62 expression, the opposite trend was observed (Fig. $2 \mathrm{H}$ ). Similar results were obtained by RT-qPCR (Fig. 2E, G and I).

Long-term culture of HK-2 cells in HG activates Notch-1/Hes-1 signaling. The expression levels of key proteins in the Notch-1/Hes-1 pathway were moderately expressed in the HK-2 cells (Fig. 3A). At $48 \mathrm{~h}$, there was significant activation of the Notch-1/Hes-1 pathway in the HK-2 cells cultured in NG + M compared with the NG group. Following a phase of inhibition, the Notch-1/Hes-1 pathway was significantly activated in the cells stimulated with HG for $48 \mathrm{~h}$ (Fig. 3B and D). Similar to the results obtained by western blot analysis, HG upregulated 

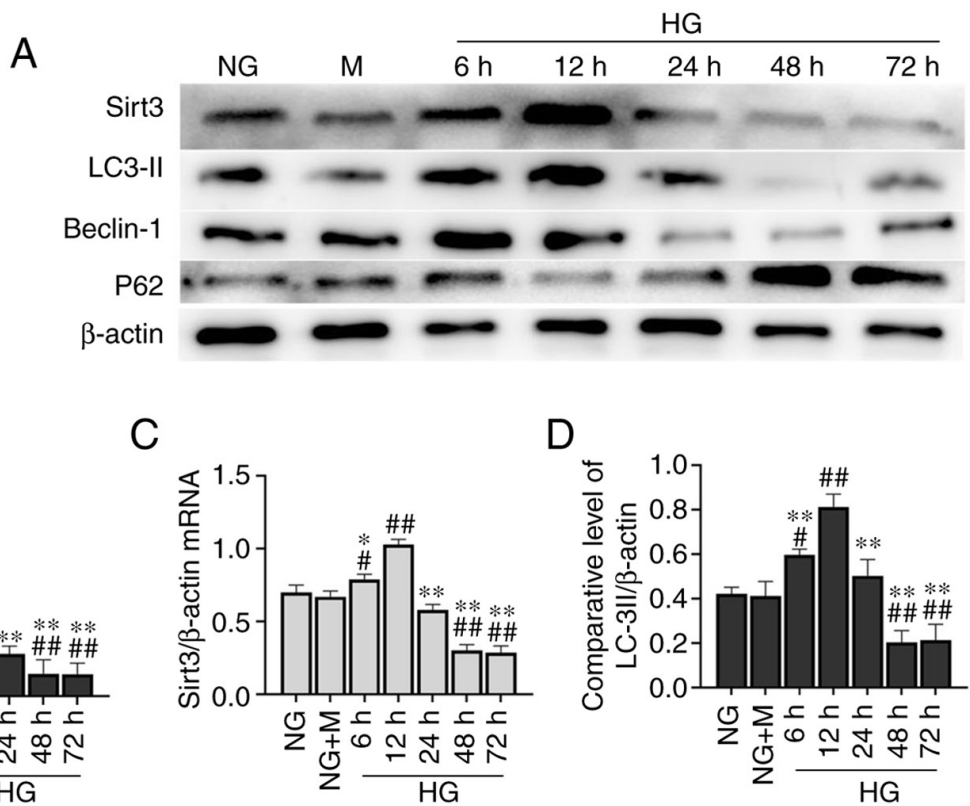

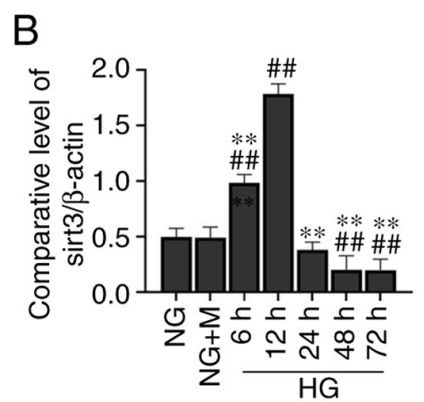

$\mathrm{F}$

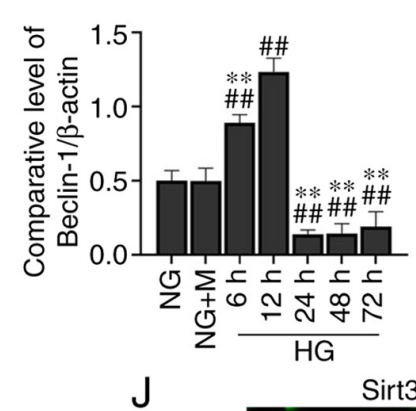

C

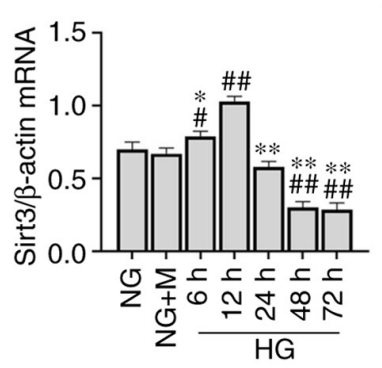

$\mathrm{G}$

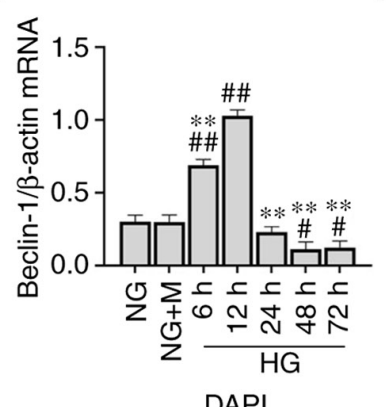

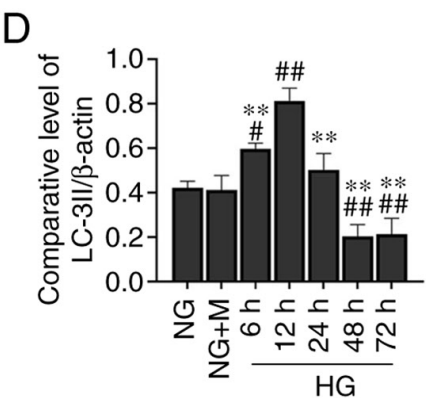

$\mathrm{H}$

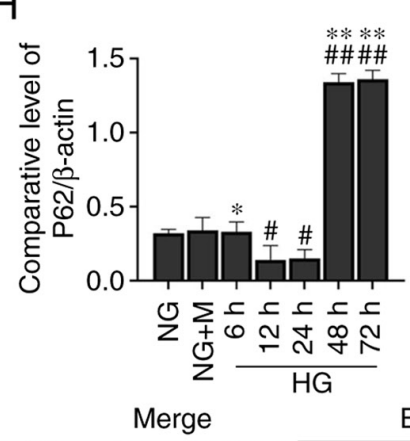

E

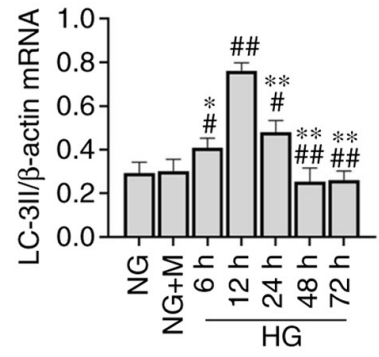

I

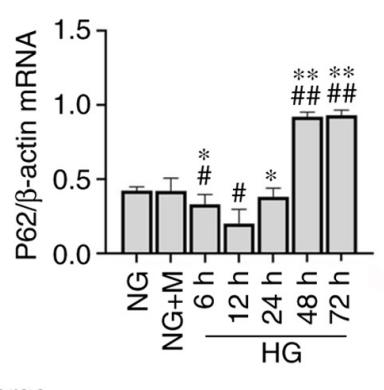

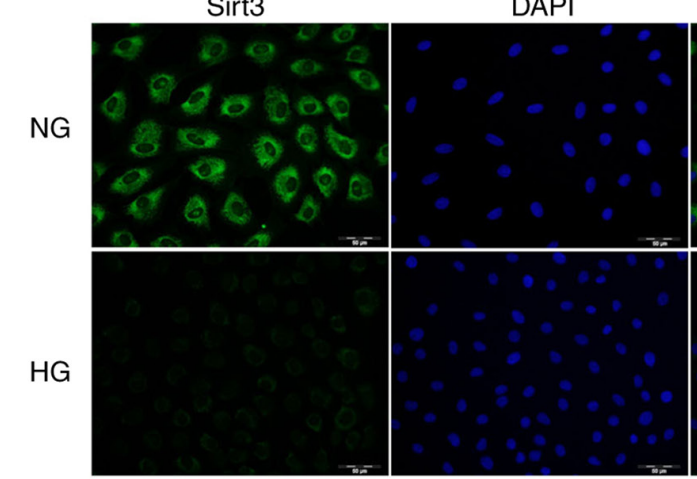

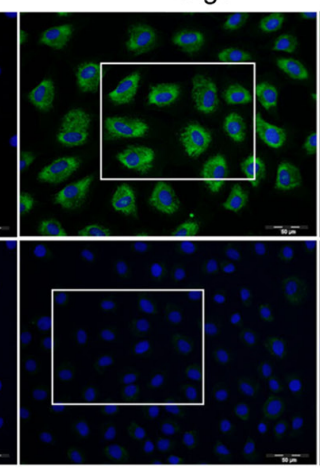

Enlarge
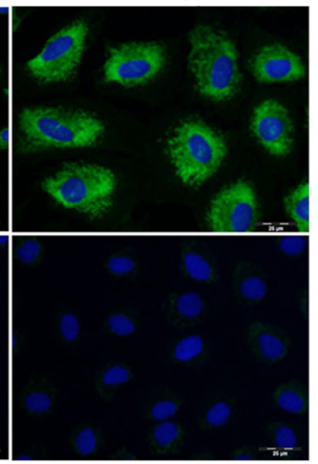

Figure 2. Long-term culture of HK-2 cells in HG inhibits expression of Sirt3 and autophagy. (A) Representative western blots of proteins examined. Expression levels of (B) Sirt3, (D) LC-3II, (F) Beclin-1 and (H) p62 were determined by western blotting. $\beta$-actin was used as an internal control. mRNA expression levels of (C) Sirt3, (E) LC-3II, (G) Beclin-1 and (I) p62 were determined by reverse transcription-quantitative PCR. (J) Effects of HG on the expression of Sirt3 were determined by immunofluorescence staining [scale bar, $50 \mu \mathrm{m}$; the enlarged image is a larger scale image of the area in the merged image represented by the white box (scale bar, $25 \mu \mathrm{m}$ )]. Sirt3 (green) was defined by staining cells with anti-Sirt3 antibody. DAPI staining (blue) was used to determine the position of the nucleus. ${ }^{\#} \mathrm{P}<0.05$ and ${ }^{\# \#} \mathrm{P}<0.01$ vs. NG; ${ }^{*} \mathrm{P}<0.05$ and ${ }^{* *} \mathrm{P}<0.01$ vs. HG at $12 \mathrm{~h}$. NG, normal glucose (5.5 mM glucose); M, mannitol (5.5 mM glucose + $24.5 \mathrm{mmol} / 1$ mannitol); HG, high glucose (30 mM glucose); Sirt3, sirtuin3.

the expression of Notch-1/Hes-1 at the transcriptional level at $48 \mathrm{~h}$ (Fig. 3C and E).

Sirt3 activates autophagy in HG-stimulated HK-2 cells. As shown in Fig. 4D-J, HG significantly decreased Beclin-1 and LC-3II expression, whereas it significantly increased p62 expression compared with the HK-2 cells in the NG group. Following transfection with pCMV-Sirt3, Sirt3 was significantly upregulated compared with the control-vector group (Fig. 4A and B), and Sirt3 was effectively activated when treated with $\mathrm{HG}+$ pCMV-Sirt3 compared with the $\mathrm{HG}+$ pCMV-vector group (Fig. 4D). Compared with NG and HG group, the overexpression of Sirt3 by transfection with pCMV-Sirt3 significantly enhanced cell viability at $48 \mathrm{~h}$ in the $\mathrm{NG}+$ pCMV-vector and $\mathrm{HG}+$ pCMV-vector groups, respectively (Fig. 4C). In addition, the expression of Beclin-1 and LC-3II was significantly elevated at the transcriptional and translational levels in the $\mathrm{HG}+$ pCMV-Sirt3 group 
A

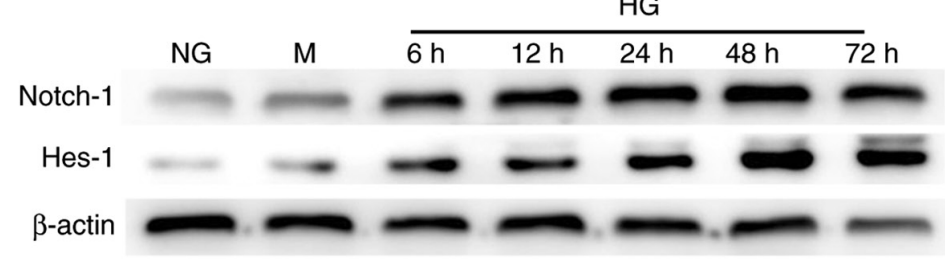

B

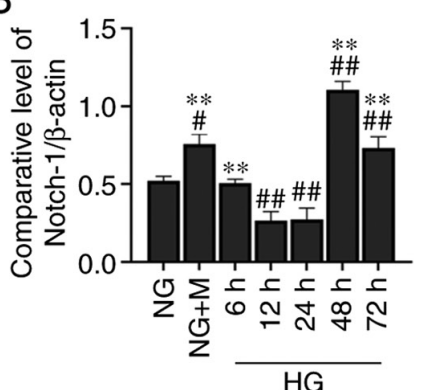

C

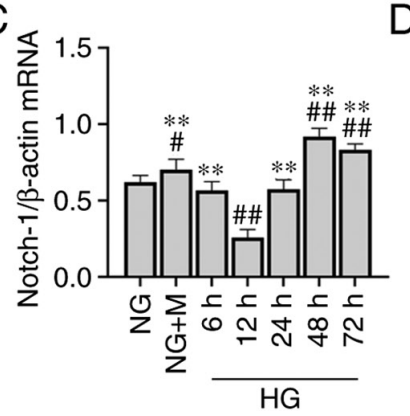

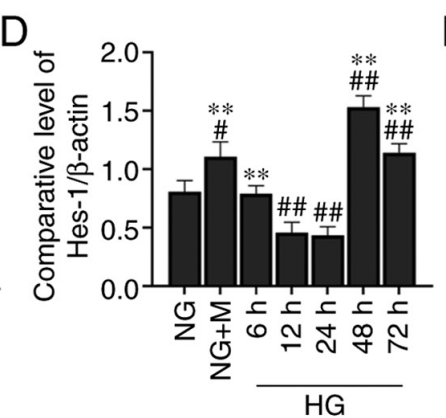

$\mathrm{E}$

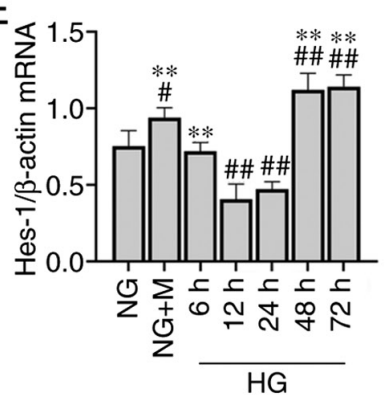

Figure 3. Long-term culture of HK-2 cells in HG activates Notch-1/Hes-1 signaling. (A) Representative western blots of proteins examined. Expression of (B and C) Notch-1 and (D and E) Hes-1 was determined by western blotting and reverse transcription-quantitative PCR. $\beta$-actin was used as an internal control. ${ }^{\#} \mathrm{P}<0.05$ and ${ }^{\# \#} \mathrm{P}<0.01$ vs. NG; ${ }^{* *} \mathrm{P}<0.01$ vs. HG at $12 \mathrm{~h}$. NG, normal glucose (5.5 mM glucose); M, mannitol (5.5 mM glucose + $\left.24.5 \mathrm{mmol} / 1 \mathrm{mannitol}\right) ; \mathrm{HG}$, high glucose (30 mM glucose); Notch-1, notch homolog 1; Hes-1, hairy and enhancer of split-1.

compared with the HG group. Moreover, pCMV-Sirt3 significantly decreased p62 expression compared with the HG group (Fig. 4E-J). These results demonstrated that Sirt3 reversed the inhibition of autophagy induced by HG. Moreover, the results presented in Fig. $4 \mathrm{~K}$ and $\mathrm{L}$ also demonstrated that $\mathrm{HG}$ inhibited the expression of Sirt3 at the transcriptional level compared with the NG group.

Sirt3 inhibits the Notch-1/Hes-1 pathway in HG-stimulated HK-2 cells. The expression of Notch-1 (Fig. 5A-C) and Hes-1 (Fig. 5A, D and E) was significantly elevated in the HK-2 cells following stimulation with $\mathrm{HG}$ for $48 \mathrm{~h}$ compared with the NG group. Nevertheless, the HG-induced activation of the Notch-1/Hes-1 pathway was attenuated when the cells were transfected with pCMV-Sirt3, which suggested that Sirt3 inhibited the Notch-1/Hes-1 pathway in the HG-stimulated HK-2 cells.

Sirt3 upregulates autophagy in $\mathrm{HK}-2$ cells via the inhibition of Notch-1/Hes-1 signaling. As illustrated in Fig. 6A-E, HG could induce activation of the Notch-1/Hes-1 signaling pathway, which was inhibited by Sirt3. Notch-1/Hes-1 pathway activation via the Notch-1/Hes-1 pathway activator, Jagged-1, significantly upregulated Notch-1/Hes-1 protein expression compared with the $\mathrm{HG}+$ pCMV-Sirt3 group. As shown in Fig. 6F, a marked decrease in Beclin-1 and LC-3II expression was observed in the HK-2 cells following stimulation with $\mathrm{HG}$ for $48 \mathrm{~h}$ (Fig. 6G-J). The accumulation of p62 was also detected in the HG-stimulated cells (Fig. 6K and L). However, the autophagic process was activated when the cells were treated with HG + pCMV-Sirt3. A significant decrease in Beclin-1 and LC-3II, and a pronounced rise in P62 was detected in the HG + pCMV-Sirt3 + Jagged-1 group compared with the $\mathrm{HG}+$ pCMV-Sirt3 group (Fig. 6G-L). These results indicated that Sirt3 positively regulated autophagy via the inhibition of the Notch-1/Hes-1 pathway.

\section{Discussion}

Autophagy is a basic process of physiological metabolism that functions to remove impaired organelles and protein aggregates, resulting in the recycling and remobilization of nutrients (21). The dysfunction of the process of autophagy has been reported to be an important pathogenic mechanism and it plays a role in the occurrence and development of several diseases (22). Under HG stress conditions, the impairment of autophagy predisposes nephrocytes to accumulate unfolded proteins and damaged organelles (23). The inhibition of autophagy induced by $\mathrm{HG}$ is considered to be one of the key pathogenetic factors for DN (24). Studies have suggested that the alleviation of autophagic obstruction is involved in the repair of the damage caused to renal tubular epithelial cells by hyperglycemia $(25,26)$. Although there is evidence that focused on the autophagic process in DN, the molecular mechanisms responsible for the regulation of autophagy remain unclear.

In the present study, it was found that cell viability increased during the early phase of HG stimulation. The viability of the HK-2 cells was decreased by extending the incubation time. In parallel, autophagy was suppressed in the HK-2 cells. The inhibitory effect was most evident at $48 \mathrm{~h}$ and later time points. A number of theoretical hypotheses have been proposed to explain the molecular pathways regulating autophagy in the diabetes-induced damage to renal tubular epithelial cells (24). Among these, Sirt1, which has been implicated as a nicotinamide adenine dinucleotide-dependent deacetylase, has been reported to be a positive regulator of autophagy (27). Sirt1 serves as a potential renoprotective factor. The low expression of Sirt1 has been shown to lead to diabetic renal injury via the negative regulation of autophagy (24). Sirt1 and Sirt3 belong to the same family and can both mediate the regulation of autophagy. In the present study, HG decreased Sirt3 expression in the HK-2 cells. To date, at least to the best of 
A

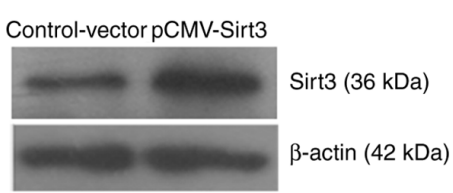

$\mathrm{B}$

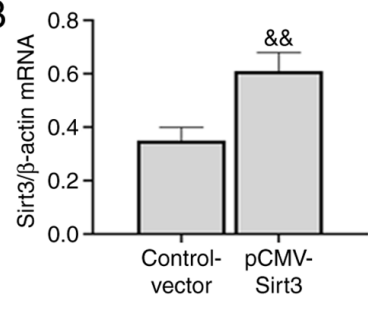

C

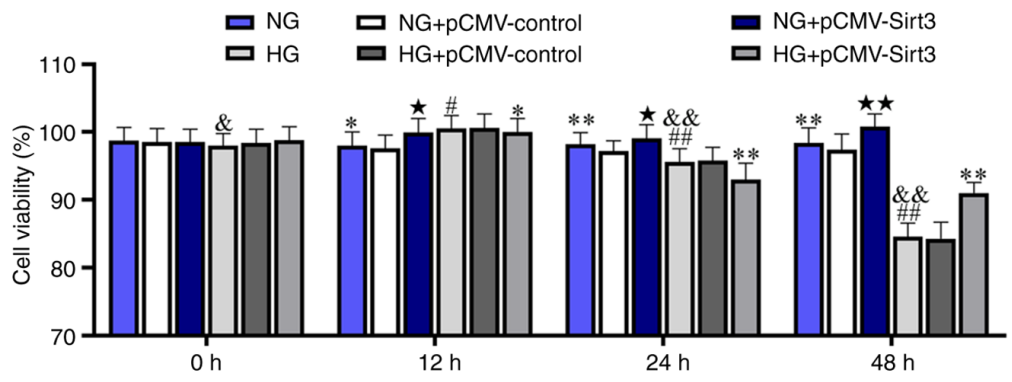

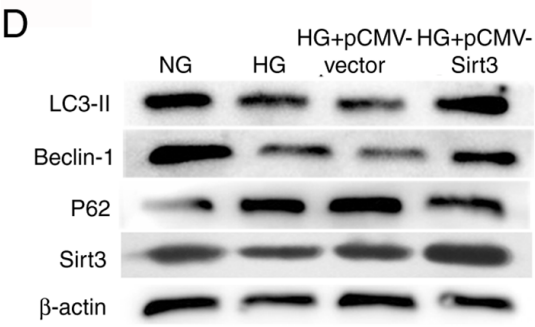

G
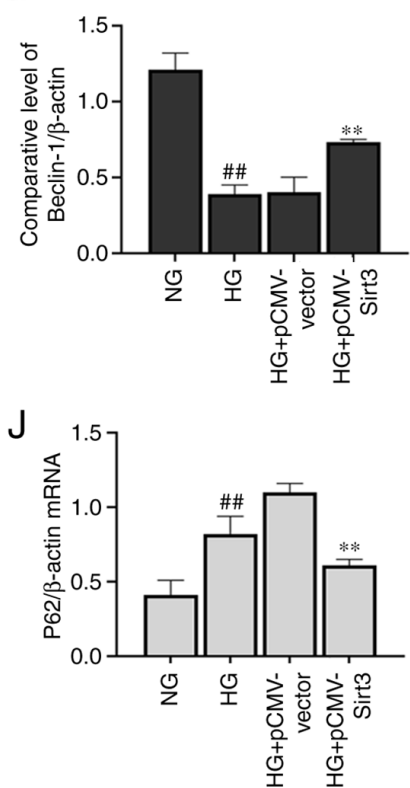

$\mathrm{E}$

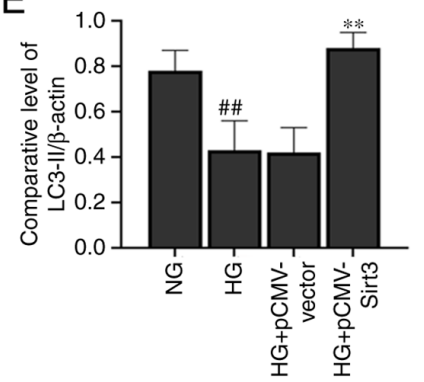

$\mathrm{H}$

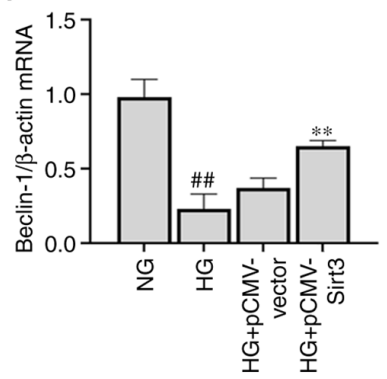

K

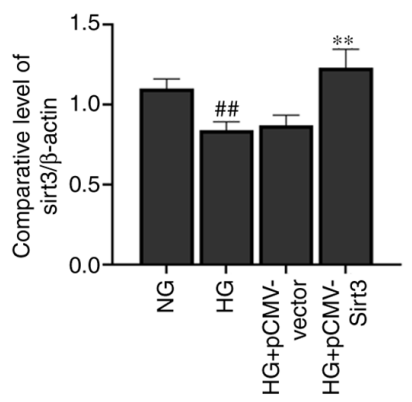

$\mathrm{F}$

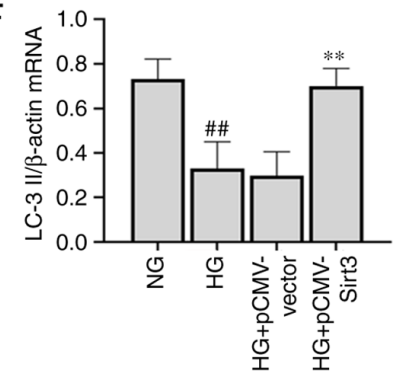

I
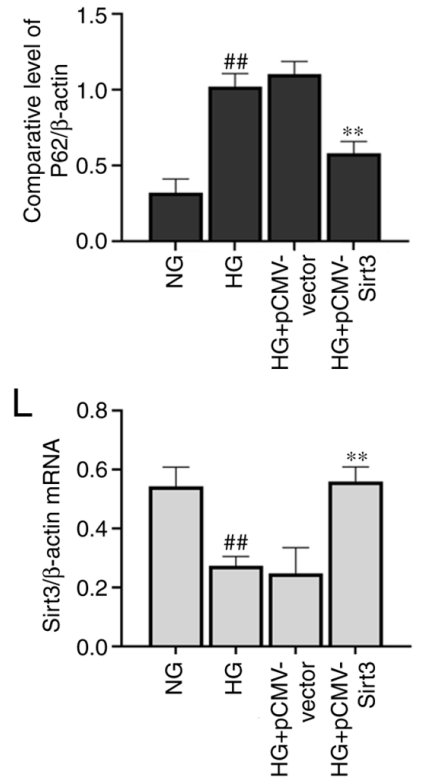

Figure 4. Sirt3 activates autophagy in HG-stimulated HK-2 cells. (A) Western blot analysis and (B) RT-qPCR were used to detect the transfection efficiency of the overexpression plasmid in HK-2 cells. ${ }^{\&}{ }^{\&} \mathrm{P}<0.01$ vs. control-vector. (C) Effect of Sirt3 on cell viability. ${ }^{*} \mathrm{P}<0.05$ and ${ }^{* *} \mathrm{P}<0.01$ vs. HG; ${ }^{*} \mathrm{P}<0.05$ and ${ }^{\# \#} \mathrm{P}<0.01$ vs. HG at $0 \mathrm{~h} ;{ }^{*} \mathrm{P}<0.05$ and ${ }^{* *} \mathrm{P}<0.01 \mathrm{vs}$. NG; ${ }^{\&} \mathrm{P}<0.05$ and ${ }^{\&} \& \mathrm{P}<0.01$ vs. HG at $12 \mathrm{~h}$. (D) Representative western blots of proteins examined. Expression levels of (E and F) LC-3II, (G and H) Beclin-1, (I and J) p62 and (K and L) Sirt3 were determined by western blot analysis and RT-qPCR. $\beta$-actin was used as an internal control. ${ }^{\# \#} \mathrm{P}<0.01$ vs. NG; ${ }^{* *} \mathrm{P}<0.01$ vs. HG. NG, normal glucose (5.5 mM glucose); HG, high glucose (30 mM glucose); RT-qPCR, reverse transcription-quantitative PCR; Sirt3, sirtuin3.

our knowledge, there is no in-depth study available on the effects of HG on Sirt3. The mechanisms through which Sirt3 functions in response to environmental signals remain to be determined. Previously, in animal experiments, Sirt3 was shown to ameliorate autophagy dysfunction in acute tubular cell injury, resulting in the upregulation of LC-3II and Beclin-1 expression (11). Additionally, a previous in vitro study revealed that Sirt 3 attenuated the inhibition of autophagy induced by 
A

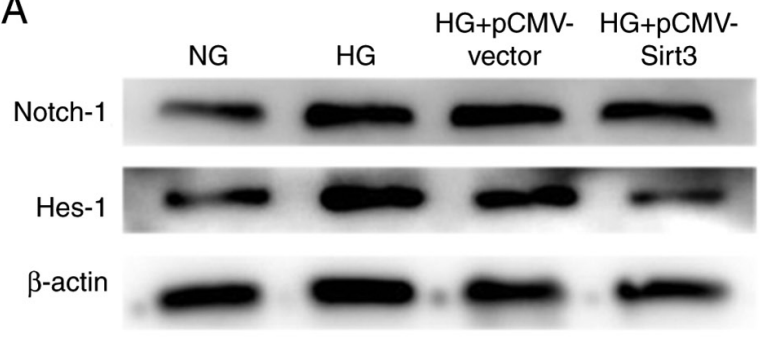

B

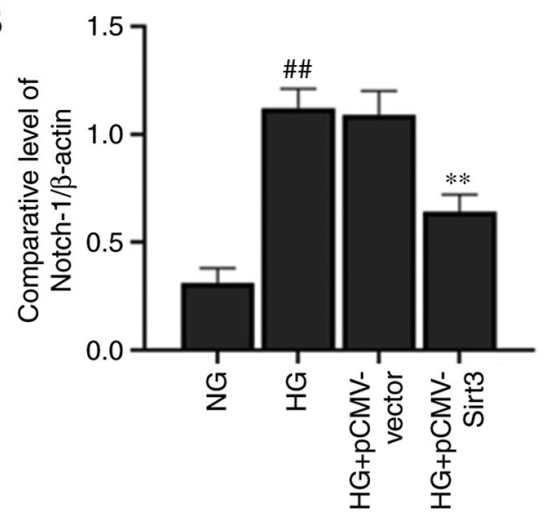

C

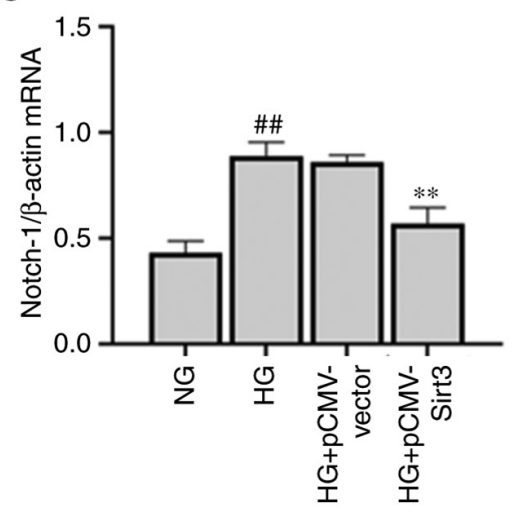

D

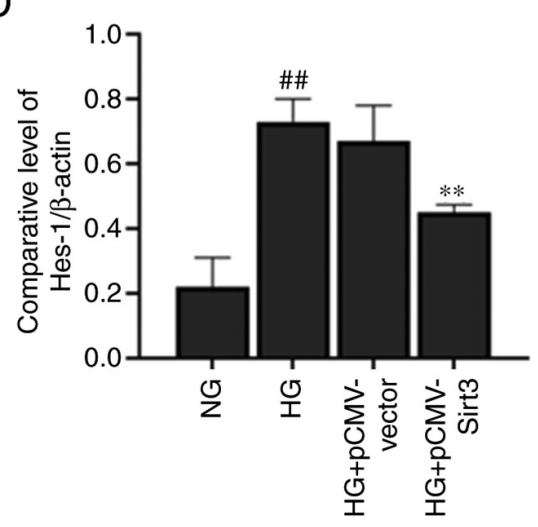

E

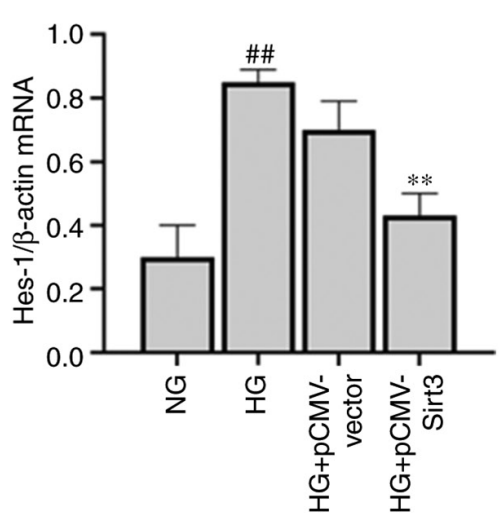

Figure 5. Sirt3 inhibits the activation of the Notch-1/Hes-1 pathway in HG-stimulated HK-2 cells. (A) Representative western blots of proteins examined. Expression of (B and C) Notch-1 and (D and E) Hes-1 were determined by western blot analysis and reverse transcription-quantitative PCR. $\beta$-actin was used as an internal control. ${ }^{\# \#} \mathrm{P}<0.01$ vs. NG; ${ }^{* *} \mathrm{P}<0.01$ vs. HG. NG, normal glucose (5.5 mM glucose); HG, high glucose (30 mM glucose); Sirt3, sirtuin3; Notch-1, notch homolog 1; Hes-1, hairy and enhancer of split-1.

high levels of oxalic acid in renal tubular epithelial cells; this led to the suppression of cell apoptosis and necrosis, as well as in the protection of renal function (28). In the present study, Sirt 3 activated autophagy in the HG-stimulated HK-2 cells, as evidenced by the upregulation of LC-3II and Beclin-1, and the downregulation of p62 expression, either at the transcriptional or post-transcriptional levels in the HK-2 cells. These results are in accordance with those of a similar previous study (11). However, unlike the present study, the previous study focused on renal tubular cell damage caused by sepsis rather than HG-induced renal tubular cell damage. Due to the different pathogenic mechanisms, whether the two diseases have anything in common in regulatory pathways warrants further investigation.

With regards to the exact mechanisms through which Sirt3 promoted autophagy, it has previously been reported that Sirt3 regulates the autophagic process by activating different downstream signaling pathways. Previously, in a cell model of rotenone-induced Parkinson's disease, Sirt3 was found to protect against neurodegenerative disease through the regulation of autophagy via activating the liver kinase B1/adenosine monophosphate-activated protein kinase (AMPK)/mTOR pathway (29). Additionally, studies have demonstrated that Sirt3 protects against cellar damage via AMPK-mediated autophagy $(11,30)$. The present study demonstrated that Sirt3 promoted the autophagy of HK-2 cells via the inhibition of Notch-1/Hes-1 signaling.
It is well-known that the Notch pathway is an evolutionarily conserved intercellular signaling pathway, and is involved in the course of renal development by regulating the differentiation and maturation of podocytes $(31,32)$. A previous study demonstrated that autophagy in podocytes was markedly diminished when Notch signaling was upregulated (33). However, studies on the association between the Notch pathway and autophagy in renal tubular epithelial cells are limited. In the present study, it was found that the Notch-1/Hes-1 pathway was activated in HG-stimulated HK-2cells. The promoting effects of Sirt 3 on autophagy were attenuated following the activation of the Notch-1/Hes-1 pathway. This provides further evidence that Sirt3 activated autophagy in HK-2 cells via the inhibition of Notch-1/Hes-1 pathway.

The findings presented herein are preliminary and thus, further in-depth investigations are warranted. In future experiments, the formation of autophagosomes should be traced using the autophagic flux indicator RFP-GFP-LC3 under fluorescence microscopy. Additionally, the formation of autophagosomes in different stages of integration should be confirmed by electron microscopy. Moreover, detecting changes in LC3-II and Beclin-1 levels alone does not appear sufficient for the evaluation of autophagic activity. The induction of autophagy and the accumulation of autophagosomes are not entirely representative of the activation of the autophagic pathway. For an improved evaluation of autophagic activity, more effective methods (e.g. electron microscopy 

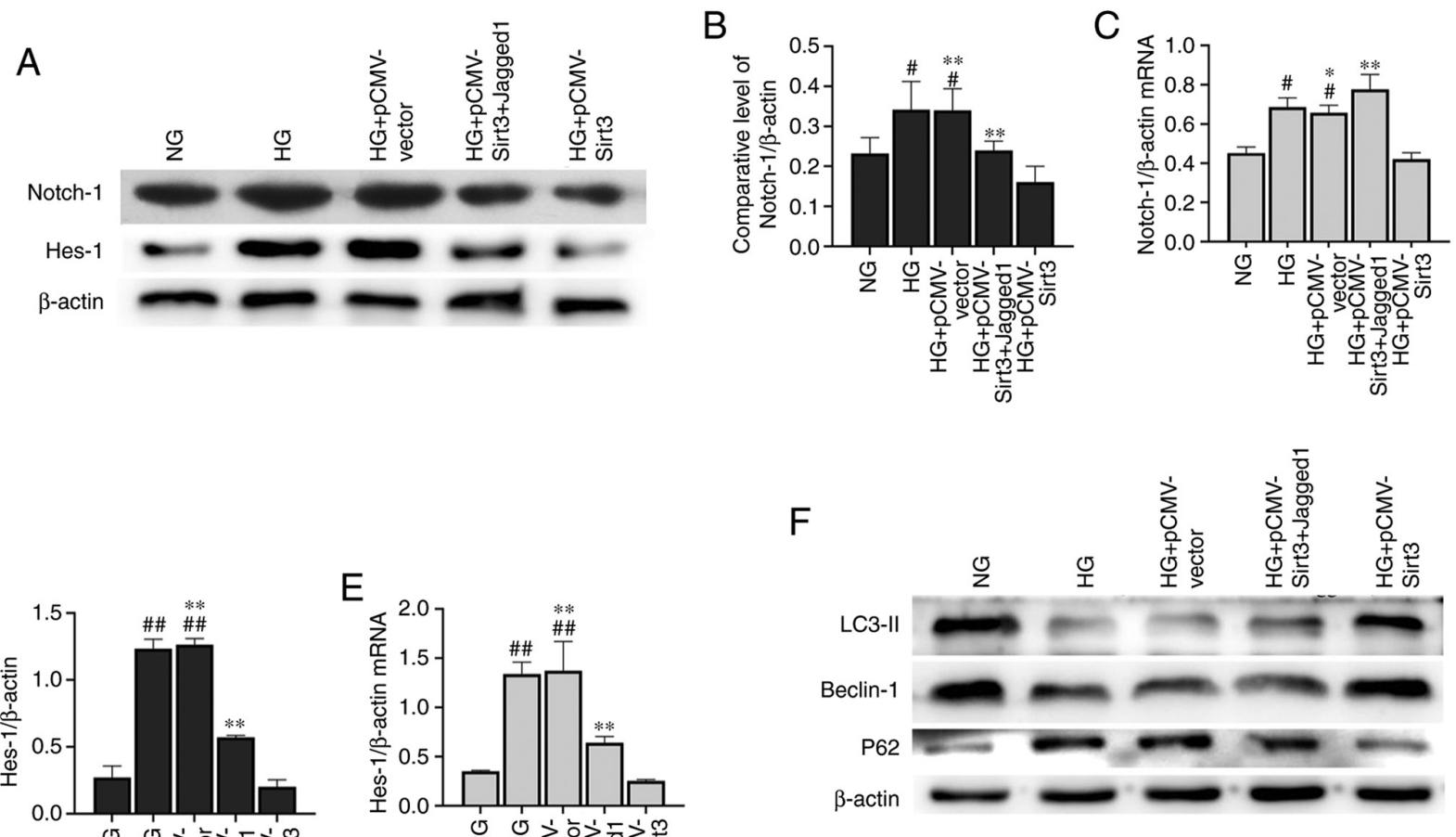
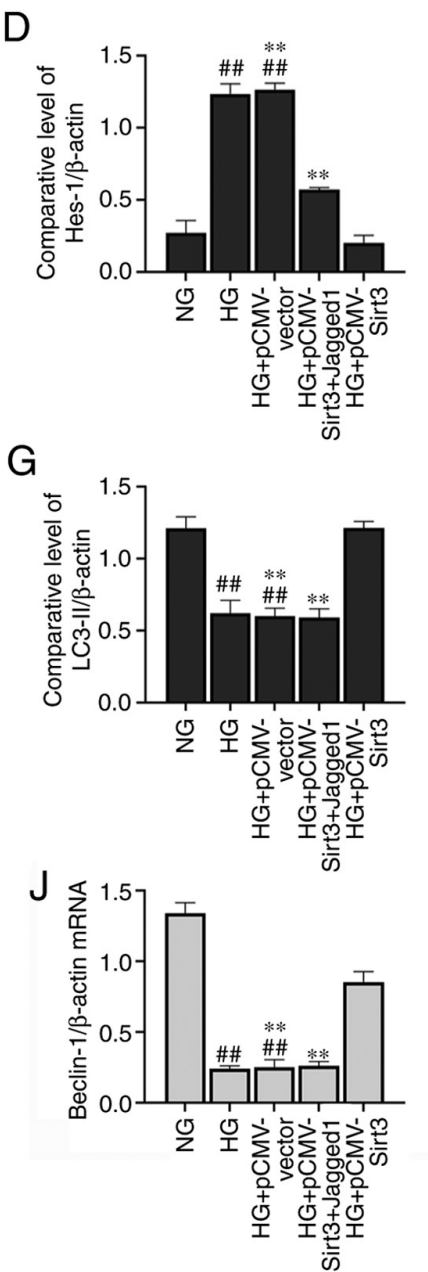

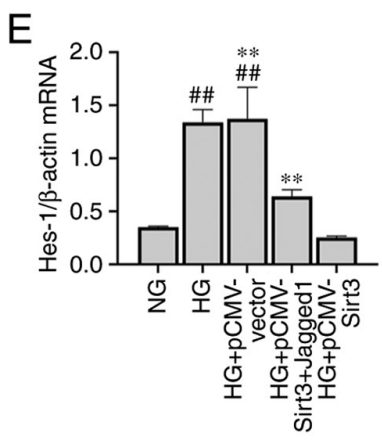

$\mathrm{H}$
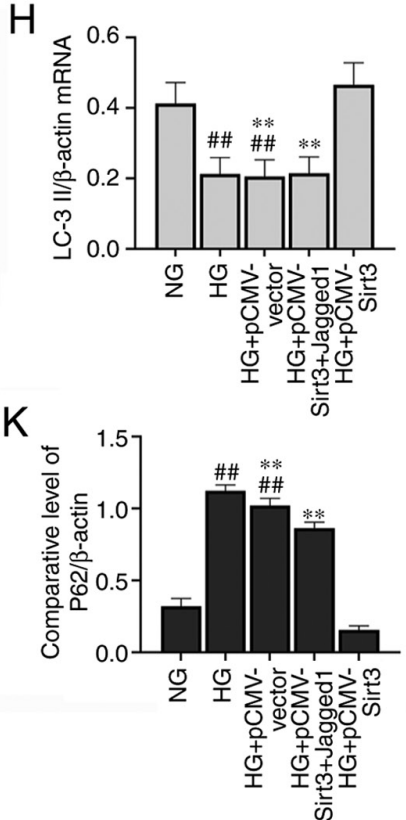

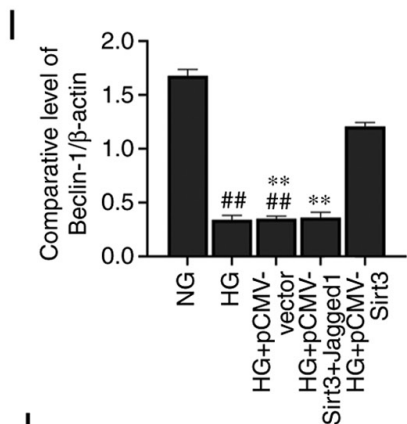

$\mathrm{L}$

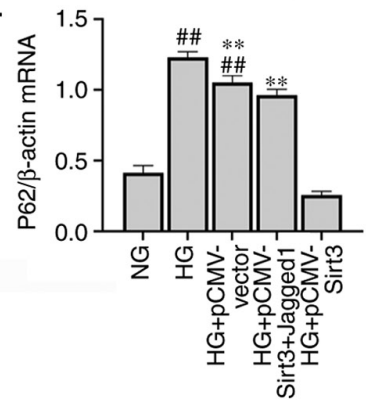

Figure 6. Sirt3 promotes the autophagy of HK-2 cells via the inhibition of Notch-1/Hes-1 signaling. (A-C) Effects of Notch-1/Hes-1 pathway activator (Jagged-1) on Notch-1 expression in HG-stimulated HK-2 cells. (D-E) Effects of Jagged-1 on Hes-1 expression in HG-stimulated HK-2 cells. (F) Representative western blots of LC-3II, Beclin-1 and p62 proteins. Expression levels of (G and H) LC-3II, (I and J) Beclin-1 and (K and L) p62 were determined by western blot analysis and reverse transcription-quantitative PCR. $\beta$-actin was used as an internal control. ${ }^{*} \mathrm{P}<0.05$ and ${ }^{\# \#} \mathrm{P}<0.01$ vs. NG; ${ }^{*} \mathrm{P}<0.05$ and ${ }^{* *} \mathrm{P}<0.01$ vs. HG + pCMV-Sirt3 group. NG, normal glucose (5.5 mM glucose); HG, high glucose (30 mM glucose); Sirt3, sirtuin3; Notch-1, notch homolog 1; Hes-1, hairy and enhancer of split-1.

and LC3-GFP imaging) should be used for detection of the degradation process.

In conclusion, the present study demonstrated that the overexpression of Sirt3 induced an increase in the levels of autophagy regulators in HK-2 cells stimulated with HG. Sirt3 activated autophagy at least partly, via the inhibition of the Notch-1/Hes-1 pathway. Thus, Sirt3 may be a viable target in the treatment of DN via the inhibition of Notch-1/Hes-1 signaling.

\section{Acknowledgements}

Not applicable.

\section{Funding}

This study was supported by the Natural Science Fund of Inner Mongolia Autonomous Region (grant no. 2019MS08064). 


\section{Availability of data and materials}

The datasets used and/or analyzed during the current study are available from the corresponding author on reasonable request.

\section{Authors' contributions}

YW, JC and YL conceived and designed the present study. YW and ZW were responsible for data analysis and performed the experiments. YW, JC and YL wrote the manuscript. YL reviewed and edited the manuscript. YW and YL confirm the authenticity of all the raw data. All authors read and approved the final manuscript.

\section{Ethics approval and consent to participate}

Not applicable.

\section{Patient consent for publication}

Not applicable.

\section{Competing interests}

The authors declare that they have no competing interests.

\section{References}

1. Warren AM, Knudsen ST and Cooper ME: Diabetic nephropathy: An insight into molecular mechanisms and emerging therapies. Expert Opin Ther Targets 23: 579-591, 2019.

2. Gilbertson DT, Liu J, Xue JL, Louis TA, Solid CA, Ebben JP and Collins AJ: Projecting the number of patients with end-stage renal disease in the United States to the year 2015. J Am Soc Nephrol 16: 3736-3741, 2005.

3. Tang C, Livingston MJ, Liu Z and Dong Z: Autophagy in kidney homeostasis and disease. Nat Rev Nephrol 16: 489-508, 2020.

4. Small DM, Bennett NC, Coombes J, Johnson DW and Gobe GC: Mitochondrial homeostasis is impeded by degradation and autophagy in oxidative stress-induced renal cell injury. Revista Española De Reumatismo Y Enfermedades Osteoarticulares 11: 67-73, 2013.

5. Kitada M, Ogura Y, Monno I and Koya D: Regulating autophagy as a therapeutic target for diabetic nephropathy. Curr Diab Rep 17: 53, 2017.

6. Zhu SY, Yao RQ, Li YX, Zhao PY, Ren C, Du XH and Yao YM: Lysosomal quality control of cell fate: A novel therapeutic target for human diseases. Cell Death Dis 11: 817, 2020

7. Guo J, Zheng HJ, Zhang W, Lou W, Xia C, Han XT, Huang WJ, Zhang F, Wang Y and Liu WJ: Accelerated kidney aging in diabetes mellitus. Oxid Med Cell Longev 2020: 1234059, 2020.

8. Lee IH, Cao L, Mostoslavsky R, Lombard DB, Liu J, Bruns NE, Tsokos M, Alt FW and Finkel T: A role for the NAD-dependent deacetylase Sirt1 in the regulation of autophagy. Proc Natl Acad Sci USA 105: 3374-3379, 2008.

9. Li R, Xin T, Li D, Wang C, Zhu H and Zhou H: Therapeutic effect of Sirtuin 3 on ameliorating nonalcoholic fatty liver disease: The role of the ERK-CREB pathway and Bnip3-mediated mitophagy. Redox Biol 18: 229-243, 2018.

10. Zhang T, Liu J, Shen S, Tong Q, Ma X and Lin L: SIRT3 promotes lipophagy and chaperon-mediated autophagy to protect hepatocytes against lipotoxicity. Cell Death Differ 27: 329-344, 2020.

11. Zhao W, Zhang L, Chen R, Lu H, Sui M, Zhu Y and Zeng L: SIRT3 protects against acute kidney injury via AMPK/mTOR-regulated autophagy. Front Physiol 9: 1526, 2018.

12. Kitada M, Kume S, Takeda-Watanabe A, Kanasaki K and Koya D: Sirtuins and renal diseases: Relationship with aging and diabetic nephropathy. Clin Sci (Lond) 124: 153-164, 2013.
13. Yu W, Gao B, Li N, Wang J, Qiu C, Zhang G, Liu M, Zhang R, Li C, Ji G and Zhang Y: Sirt3 deficiency exacerbates diabetic cardiac dysfunction: Role of Foxo3A-Parkin-mediated mitophagy. Biochim Biophys Acta Mol Basis Dis 1863: 1973-1983, 2017.

14. Chen Y, Zhang F, Wang D, Li L, Si H, Wang C, Liu J, Chen Y, Cheng $\mathrm{J}$ and $\mathrm{Lu} \mathrm{Y}$ : Mesenchymal stem cells attenuate diabetic lung fibrosis via adjusting Sirt3-mediated stress responses in rats. Oxid Med Cell Longev 2020: 8076105, 2020

15. Artavanis-Tsakonas S, Rand MD and Lake RJ: Notch signaling: Cell fate control and signal integration in development. Science 284: 770-776, 1999.

16. Yuri S, Nishikawa M, Yanagawa N, Jo OD and Yanagawa N: Maintenance of mouse nephron progenitor cells in aggregates with Gamma-secretase inhibitor. PLoS One 10: e0129242, 2015.

17. Wang Y, Li Y, Yang Z, Wang Z, Chang J, Zhang T, Chi Y, Han N and Zhao K: Pyridoxamine treatment of HK-2 human proximal tubular epithelial cells reduces oxidative stress and the inhibition of autophagy induced by high glucose levels. Med Sci Monit 25: $1480-1488,2019$

18. Jiao X, Li Y, Zhang T, Liu M and Chi Y: Role of Sirtuin3 in high glucose-induced apoptosis in renal tubular epithelial cells. Biochem Biophys Res Commun 480: 387-393, 2016.

19. Wang Z, Li Y, Wang Y, Zhao K, Chi Y and Wang B: Pyrroloquinoline quinine protects HK-2cells against high glucose-induced oxidative stress and apoptosis through Sirt3 and PI3K/Akt/FoxO3a signaling pathway. Biochem Biophys Res Commun 508: 398-404, 2019.

20. Livak KJ and Schmittgen TD: Analysis of relative gene expression data using real-time quantitative PCR and the 2(-Delta Delta C(T)) method. Methods 25: 402-408, 2001.

21. Mizushima N and Levine B: Autophagy in human diseases. $N$ Engl J Med 383: 1564-1576, 2020.

22. Jiang P and Mizushima N: Autophagy and human diseases. Cell Res 24: 69-79, 2014.

23. Kim KH and Lee MS: Autophagy-a key player in cellular and body metabolism. Nat Rev Endocrinol 10: 322-337, 2014.

24. Ding Y and Choi ME: Autophagy in diabetic nephropathy. J Endocrinol 224: R15-R30, 2015.

25. Liu WJ, Huang WF, Ye L, Chen RH, Yang C, Wu HL, Pan QJ and Liu HF: The activity and role of autophagy in the pathogenesis of diabetic nephropathy. Eur Rev Med Pharmacol Sci 22: 3182-3189, 2018.

26. Lin F: Autophagy in renal tubular injury and repair. Acta Physiol (Oxf) 220: 229-237, 2017.

27. Ng F and Tang BL: Sirtuins' modulation of autophagy. J Cell Physiol 228: 2262-2270, 2013.

28. Peng Y, Yang C, Shi X, Li L, Dong H, Liu C, Fang Z, Wang Z, Ming S, Liu M, et al: Sirt3 suppresses calcium oxalate-induced renal tubular epithelial cell injury via modification of FoxO3a-mediated autophagy. Cell Death Dis 10: 34, 2019.

29. Zhang M, Deng YN, Zhang JY, Liu J, Li YB, Su H and Qu QM: SIRT3 protects Rotenone-induced injury in SH-SY5Y cells by promoting autophagy through the LKB1-AMPK-mTOR Pathway. Aging Dis 9: 273-286, 2018.

30. Wang Y, Zhang X, Wang P, Shen Y, Yuan K, Li M, Liang W and Que H: Sirt3 overexpression alleviates hyperglycemia-induced vascular inflammation through regulating redox balance, cell survival, and AMPK-mediated mitochondrial homeostasis. J Recept Signal Transduct Res 39: 341-349, 2019.

31. Surendran K, Boyle S, Barak H, Kim M, Stomberski C, McCright B and Kopan R: The contribution of Notch1 to nephron segmentation in the developing kidney is revealed in a sensitized Notch 2 background and can be augmented by reducing Mint dosage. Dev Biol 337: 386-395, 2010.

32. Vooijs M, Ong CT, Hadland B, Huppert S, Liu Z, Korving J, van den Born M, Stappenbeck T, Wu Y, Clevers H and Kopan R: Mapping the consequence of Notch1 proteolysis in vivo with NIP-CRE. Development 134: 535-544, 2007.

33. Zheng D, Tao M, Liang X, Li Y, Jin J and He Q: p66Shc regulates podocyte autophagy in high glucose environment through the Notch-PTEN-PI3K/Akt/mTOR pathway. Histol Histopathol 35: 405-415, 2020.

This work is licensed under a Creative Commons Attribution-NonCommercial-NoDerivatives 4.0 International (CC BY-NC-ND 4.0) License. 